\title{
Vacuum-assisted mini-percutaneous nephrolithotomy: a new perspective in fragments clearance and intrarenal pressure control
}

\author{
Stefano Paolo Zanetti ${ }^{1}$ ( . Elena Lievore ${ }^{1} \cdot$ Matteo Fontana ${ }^{1} \cdot$ Matteo Turetti $^{1} \cdot$ Andrea Gallioli $^{1} \cdot$ Fabrizio Longo $^{1}$. \\ Giancarlo Albo $^{2}$. Elisa De Lorenzis ${ }^{2}$. Emanuele Montanari ${ }^{2}$
}

Received: 31 January 2020 / Accepted: 16 June 2020 / Published online: 26 June 2020

(c) The Author(s) 2020

\begin{abstract}
Purpose To describe the vacuum-assisted mini-percutaneous nephrolithotomy (vmPCNL) technique performed via the $16 \mathrm{Ch}$ ClearPetra sheath, to evaluate its outcomes and to analyze intrarenal pressure (IRP) fluctuations during surgery.

Methods Data from all consecutive vmPCNL procedures from September 2017 to October 2019 were prospectively collected. Data included patients' and stones characteristics, intra and peri-operative items, post-operative complications and stone clearance. Patients undergoing vmPCNL from March to October 2019 were submitted to IRP measurement during surgery.

Results A total of $122 \mathrm{vmPCNL}$ procedures were performed. Median stone volume was $1.92 \mathrm{~cm}^{3}$. Median operative time was $90 \mathrm{~min}$ and median lithotripsy and lapaxy time was $28 \mathrm{~min}$. Stone clearance rate was $71.3 \%$. Thirty-one (25.2\%) patients experienced post-operative complications, seven of which were Clavien 3. Postoperative fever occurred in nine (7.4\%) patients and one $(0.8 \%)$ needed a transfusion. No sepsis were observed. IRPs were measured in 22 procedures. Mean IRP was 15.3 $\mathrm{cmH}_{2} \mathrm{O}$ and median accumulative time with IRP $>40.78 \mathrm{cmH}_{2} \mathrm{O}$ (pyelovenous backflow threshold) was $28.52 \mathrm{sec}$. Maximum IRP peaks were reached during the surgical steps when aspiration is closed (mainly pyelograms), whereas during lithotripsy and suction-mediated lapaxy, the threshold of $40.78 \mathrm{cmH}_{2} \mathrm{O}$ was overcome in three procedures.

Conclusions vmPCNL is a safe procedure with satisfactory stone clearance rates. Mean IRP was always lower than the threshold of pyelo-venous backflow and the accumulative time with IRP over this limit was short in most of the procedures. During lithotripsy and vacuum-mediated lapaxy, IRP rarely raised over the threshold.
\end{abstract}

Keywords Percutaneous nephrolithotomy $\cdot$ Intrarenal pressure $\cdot$ Urolithiasis $\cdot$ Endourology $\cdot$ Vacuum-assisted mini-PCNL

\section{Introduction}

Percutaneous nephrolithotomy (PCNL) is the standard of care for large kidney stones [1], but complications like fever and bleeding can represent a major concern [2]. To reduce the morbidity associated with this procedure, miniaturized

Stefano Paolo Zanetti

stefano.p.zanetti@gmail.com

1 Department of Urology, Foundation IRCCS Ca' Granda Ospedale Maggiore Policlinico, Via della Commenda 15, 20122 Milan, Italy

2 Department of Urology, Foundation IRCCS Ca' Granda Ospedale Maggiore Policlinico, Department of Clinical Sciences and Community Health, University of Milan, Milan, Italy
PCNL systems were developed [3-6]. However, these systems present some limitations such as a more difficult stone fragments retrieval, a smaller visual field, longer operative times (OT) and higher intrarenal pressures (IRPs) [7, 8]. In particular, IRP higher than $30 \mathrm{mmHg}\left(40.78 \mathrm{cmH}_{2} \mathrm{O}\right)$ has been proven to cause pyelovenous backflow [9], potentially leading to infectious complications [10]. To overcome these limitations, mini-PCNL systems provided with aspirating sheaths have been introduced. The real-time suction of irrigation fluid, stone fragments and blood throughout the procedure may lower IRP, ameliorate visibility and quicken the procedure. The aim of this study is to describe the vacuumassisted mini-PCNL (vmPCNL) technique performed using the 16 Ch Clear Petra nephrostomic sheath and to evaluate its clinical outcomes. Ultimately, we aim to analyze IRPs 
profile during surgery and to identify the procedural steps during which IRP may rise the most.

\section{Patients and methods}

Data from all consecutive vmPCNL procedures performed at our academic referral stone center from September 2017 to October 2019 were prospectively collected. The indication to vmPCNL was given in all cases when PCNL was planned, except in case of large staghorn stones, for which standard PCNL (22-24 Ch) was indicated. In case of multiple stones allocated in different calyces with a significant total stone burden, for which a single standard-tract PCNL might not be surely efficacious, a multi-staged vmPCNL procedure was pre-operatively planned in order to reduce the operative time of the single procedures. Collected data concerned patients' and stones' characteristics, intra- and peri-operative items, post-operative complications, stone clearance and need of retreatment. Comorbidities were graded according to the Charlson Comorbidity Index [11]. Intraoperative items included number and location of the percutaneous tracts, fragments retrieval modality, lithotripsy and lapaxy time (LT) (from first laser activation to the end of fragments retrieval), exit strategy, operative time (OT) (from the beginning of ureteric catheter placement to the exit strategy) and intraoperative complications. Post-operative items included hemoglobin drop, need for transfusions, nephrostomy indwelling time and length of hospital stay. Post-operative complications were graded according to the PCNL-adjusted Clavien Score [12]. Sepsis was defined according to the Sequential [Sepsis-related] Organ Failure Assessment (SOFA) score criteria [13]. Stone clearance was defined as the absence of residual fragments larger than $4 \mathrm{~mm}$ at the CT scan or ultrasound (US) performed 1-3 months after surgery. All patients underwent pre-operative urographic $\mathrm{CT}$ scan and urine culture. In case of negative culture, one-shot antibiotic prophylaxis was administered; in case of positive culture, antibiotic targeted therapy was started 3-5 days before surgery. Stone volume was measured using the ellipsoid formula ( $a \times b \times c \times \pi / 6)$.

\section{Armamentarium}

The vmPCNL procedures were performed using a $12 \mathrm{Ch}$ nephroscope (MIP set, Karl Storz) and a 16 Ch Clear Petra disposable nephrostomic sheath (Well Lead Medical Co.). This sheath is externally plugged to prevent the medium from flowing out and it is equipped with a lateral oblique arm connected to the central vacuum system (Fig. 1). This allows the continuous aspiration of stone powder and irrigation fluid beside the scope during lithotripsy. Larger fragments are retrieved by drawing back the nephroscope inside the sheath as far as the internal opening of the lateral aspiration arm, wide enough to allow the passage of stones as large as $8 \mathrm{~mm}$. Aspirated stones are collected in a dedicated plastic bottle. Irrigation is provided by a saline gravity bag allocated $1.5 \mathrm{~m}$ above the kidney level. The aspiration
Fig. 1 a The ClearPetra set (Well Lead Medical Co., Ltd., China) is composed of a Y-shaped nephrostomic sheath connected, by means of an aspiration tube, to a stone collection bottle $(200 \mathrm{ml})$, which is in turn linked to the central vacuum system. b A valve on the stone collection bottle regulates the aspiration pressure. $\mathbf{c}$ A vent on the lateral arm of the sheath can be closed with a finger to increase aspiration pressure during lapaxy. In the meantime the nephroscope $\mathbf{d}$ is slowly drawn back until the red stripe on the sheath, thus allowing fragments to fall in the lateral arm of the sheath and in the stone collection bottle
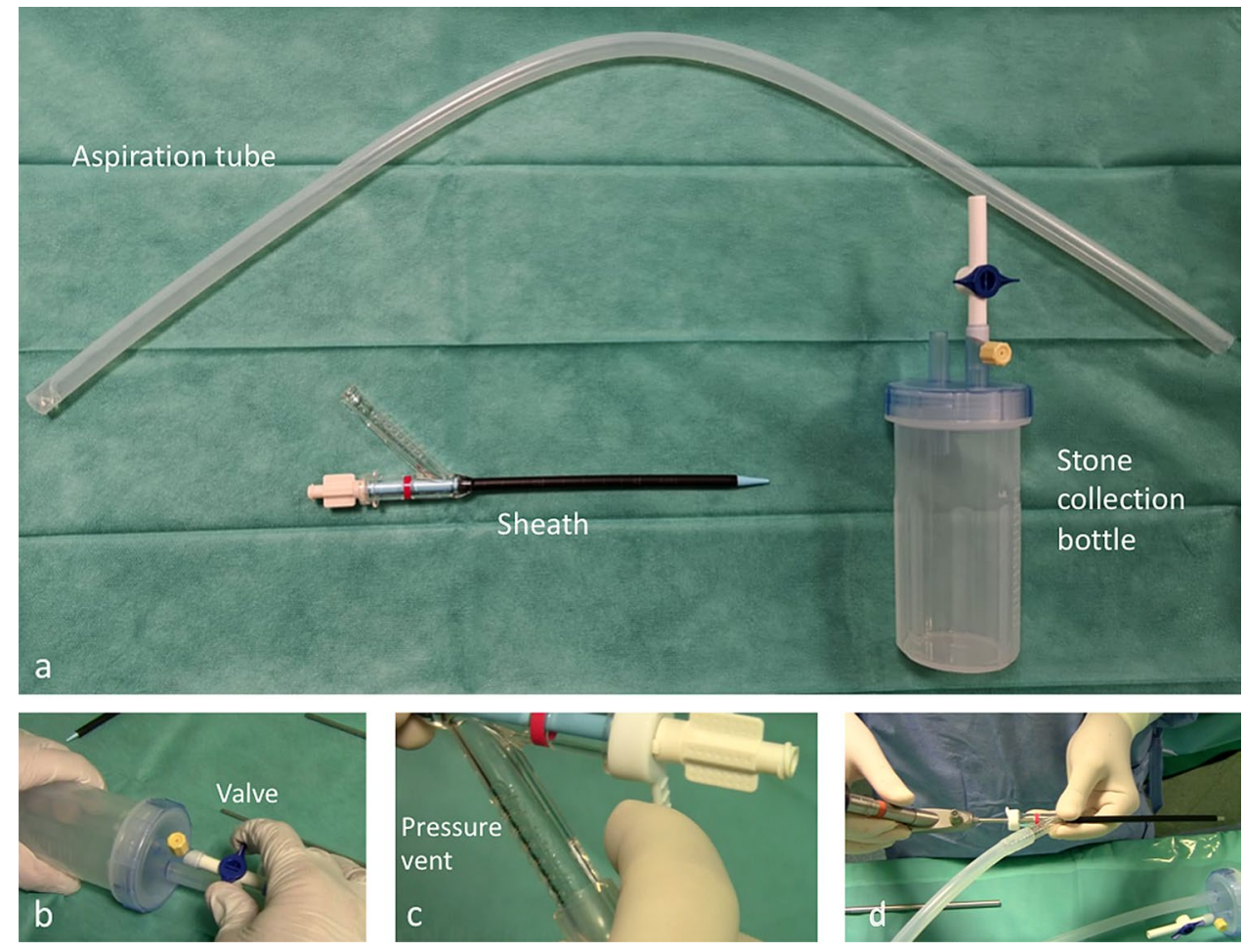
pressure can be regulated throughout the procedure according to surgical needs as shown in Fig. 1, in particular it can be enhanced to ameliorate visibility in the presence of stone powder or blood and while withdrawing the nephroscope inside the sheath to extract stone fragments.

\section{Surgical technique}

The procedure starts by placing a ureteric catheter in the renal pelvis and obtaining a retrograde pyelogram. With the patient in the semi-supine Valdivia position, renal puncture is performed under fluoroscopic/ultrasonographic control. Tract dilation is performed one-shot, using the $16 \mathrm{Ch}$ Clear Petra sheath assembled with its stylet. After introducing the nephroscope in the renal cavities, the aspiration valve is switched on. Lithotripsy is performed via a $550 \mu \mathrm{m}$ Holmium: YAG laser fiber. Stone fragments are real-time evacuated during lithotripsy through suction; a basket can be employed to catch fragments in calyces not aligned with the aspirating sheath, only reachable with a flexible scope. A nephrostomy tube is usually placed as exit strategy.

\section{Intrarenal pressure measurement}

The subgroup of continuous patients undergoing vmPCNL from March to October 2019 were submitted to IRP measurement. After zero adjustment, IRP was measured throughout the procedures, with a frequency of 50 measurements per second, through the open-end ureteric catheter in the renal pelvis, whose external end was connected to a pressure transducer of the urodynamic machine (Medtronic, Duet ${ }^{\circledR}$ Multi-P). Basal IRP was recorded before renal puncture. Mean and maximum IRPs and the accumulative time with IRP $>40.78 \mathrm{cmH}_{2} \mathrm{O}$ were calculated for every procedure. The procedures were split in different surgical steps to analyze IRP fluctuations along surgery.

\section{Statistical analysis}

Data collection and analysis were performed using the statistical software SPSS 25.0.0.1 (CIBM Corp.). Mean and standard deviation (SD) and median and interquartile ranges (IQRs) were reported for continuous variables and proportions and percentages for categorical variables.

\section{Results}

A total of $122 \mathrm{vmPCNL}$ procedures were performed on 119 renal units of 115 patients. Patients' and stones' baseline characteristics are reported in Table 1. Seventy-four (60.7\%) patients had multiple stones and the median stone volume was $1.92 \mathrm{~cm}^{3}$. Surgery-related variables and post-operative
Table 1 Patients' and stones' baseline characteristics

\begin{tabular}{|c|c|}
\hline & Total: 122 \\
\hline \multicolumn{2}{|l|}{$\operatorname{Sex}, N^{\circ}(\%)$} \\
\hline Male & $74(60.6)$ \\
\hline Female & $48(39.4)$ \\
\hline Mean age $( \pm S D)$, years & $55.2( \pm 14.5)$ \\
\hline \multicolumn{2}{|l|}{$\mathrm{BMI}, N^{\circ}(\%)$} \\
\hline$<18.5$ & $5(4.1)$ \\
\hline $18.5-24.9$ & $60(49.2)$ \\
\hline $25-29.9$ & $47(38.5)$ \\
\hline$\geq 30$ & $10(8.2)$ \\
\hline \multicolumn{2}{|l|}{ Comorbidities, $N^{\circ}(\%)$} \\
\hline Diabetes & $17(13.9)$ \\
\hline Hypertension & $32(26.2)$ \\
\hline Cardiovascular disease & $12(9.8)$ \\
\hline Chronic kidney disease & $3(2.5)$ \\
\hline Inflammatory bowel disease & $5(4.1)$ \\
\hline Hyperparathyroidism & $3(2.5)$ \\
\hline Haemorrhagic diathesis & $2(1.6)$ \\
\hline Recurrent urinary tract infections & $22(18.3)$ \\
\hline \multicolumn{2}{|l|}{ Drugs, $N^{\circ}(\%)$} \\
\hline Anticoagulants & $4(3.3)$ \\
\hline Antiplatelets & $16(13.1)$ \\
\hline \multicolumn{2}{|c|}{ American Society of Anesthesiologists score, $N^{\circ}(\%)$} \\
\hline 1 & $22(18)$ \\
\hline 2 & $79(64,8)$ \\
\hline 3 & $21(17.2)$ \\
\hline \multicolumn{2}{|l|}{ Charlson Comorbidity Index, $N^{\circ}(\%)$} \\
\hline 0 & $88(72.1)$ \\
\hline 1 & $19(15.6)$ \\
\hline 2 & $8(6.5)$ \\
\hline 3 & $3(2.5)$ \\
\hline 4 & $3(2.5)$ \\
\hline 5 & $1(0.8)$ \\
\hline Urinary tract abnormalities, $N^{\circ}(\%)$ & $6(4,9)$ \\
\hline Renal malrotation & $2(1.6)$ \\
\hline Horseshoe kidney & $1(0.8)$ \\
\hline Ectopic kidney & $1(0.8)$ \\
\hline Duplex ureter & $2(1.6)$ \\
\hline Single kidney, $N^{\circ}(\%)$ & $3(2.5)$ \\
\hline Skeletal abnormalities, $N^{\circ}(\%)$ & $2(1.6)$ \\
\hline Previous homolateral stone treatment, $N^{\circ}(\%)$ & $61(50)$ \\
\hline \multicolumn{2}{|l|}{ Stone number, $N^{\circ}(\%)$} \\
\hline Single & $48(39.3)$ \\
\hline Multiple & $74(60.7)$ \\
\hline Median total stone volume (IQR), $\mathrm{cm}^{3}$ & $1.92(1-3.1)$ \\
\hline Median mean stone density (IQR), HU & $850(550-998)$ \\
\hline \multicolumn{2}{|l|}{ Stone location, $N^{\circ}(\%)$} \\
\hline Lower calyx & $15(12.3)$ \\
\hline Middle calyx & $6(4.9)$ \\
\hline Renal pelvis & $24(19.6)$ \\
\hline Upper calyx & $4(3.3)$ \\
\hline
\end{tabular}


Table 1 (continued)

\begin{tabular}{ll}
\hline & Total: 122 \\
\hline Multiple locations & $73(59.9)$ \\
Pre-operative positive urine culture, $N^{\circ}(\%)$ & $19(15.6)$ \\
Stone composition, $N^{\circ}(\%)$ & \\
Calcium Oxalate Monohydrate & $38(31.1)$ \\
Calcium Oxalate Dihydrate & $26(21.3)$ \\
Uric acid & $25(20.5)$ \\
Calcium Carbonate + Calcium Oxalate & $10(8.2)$ \\
Calcium Carbonate & $7(5.7)$ \\
Calcium Oxalate Mono + Dihydrate & $5(4.1)$ \\
Calcium Phosphate & $4(3.3)$ \\
Cystine & $4(3.3)$ \\
Struvite & $3(2.5)$ \\
\hline
\end{tabular}

outcomes are shown in Table 2. Eleven procedures (9\%) were performed with multiple Clear Petra tracts. A basket was employed for fragments retrieval in 44 (36.1\%) procedure in addition to suction. The median OT was $90 \mathrm{~min}$ and the median LT was 28 min. A total of 31 (25.2\%) patients experienced post-operative complications, seven out of which were Clavien 3: three cases of renal colic managed by double $\mathrm{J}$ placement; three cases of bleeding, one requiring bladder irrigations and two needing angioembolization; and one case of colon perforation managed by temporary colostomy. One $(0.8 \%)$ patient needed a blood transfusion and nine $(7.4 \%)$ patients experienced fever managed by antibiotics (Clavien 2). No case of sepsis was observed. Stone clearance was obtained in eighty-seven (71.3\%) patients. Out of the 35 patients $(28.7 \%)$ who did not reach the stone clear status, nine $(7.4 \%$ of the total) were retreated within the first 6 month of follow-up for residual fragments larger than $10 \mathrm{~mm}$ : seven of them underwent a second PCNL procedure, in a pre-operatively planned multi-staged procedure, and two were submitted to retrograde intrarenal surgery. All of them resulted stone clear after the second procedure. The remaining 26 (21.3\% of the total) patients had non-obstructing residual fragments smaller than $10 \mathrm{~mm}$ and were planned to undergo follow-up imaging.

Data related to IRPs are reported in Table 3a. Twenty-two patients were included in the IRP measurement sub-cohort. The mean IRP during the procedures was $15.38 \mathrm{cmH}_{2} \mathrm{O}$. In all procedures but three, peaks over the threshold of 40.78 $\mathrm{cmH}_{2} \mathrm{O}$ were registered. Median accumulative time with IRP $>40.78 \mathrm{cmH}_{2} \mathrm{O}$ was 28.52 s. During lithotripsy and suction-mediated lapaxy, the mean IRP was $13.29 \mathrm{cmH}_{2} \mathrm{O}$ and the threshold of $40.78 \mathrm{cmH}_{2} \mathrm{O}$ was overcome in three procedures. Maximum peaks were reached during pyelograms in thirteen (59\%) procedures, during nephroscopy with closed aspiration in seven $(32 \%)$ and during puncture in two (9\%). IRP values during the different surgical steps are reported in Table $3 \mathrm{~b}$. Graphics representing the complete IRP profile during the 22 procedures are available as Online Resource. Two patients undergoing IRP measurement experienced Clavien 2 post-operative complications: one case of bleeding needing transfusion and one case of fever.

\section{Discussion}

To the best of our knowledge, this is the first study describing the vmPCNL technique performed with the Clear Petra nephrostomic sheath and analyzing IRPs profile during this procedure. The most described aspiration-assisted mini-PCNL system is the super-mini-PCNL (SMP) [14, 15], consisting of a fiberoptic nephroscope and a Y-shaped nephrostomic sheath connected to the aspiration. The Clear Petra sheath employs the same principles of SMP. One of the main advantages of these sets is that, while working with the aspirating sheath in close contact with the stone, the vacuum keeps the calculus in position during lithotripsy and directly attract fragments, thus preventing their scattering. In the first series of 141 patients treated with SMP [14], Zeng and colleagues reported a stone clearance rate of $90.1 \%$, a fever rate of $11.3 \%$ and no transfusions. In our series, we obtained stone clearance in $71.3 \%$ of the patients. This result may be considered satisfactory for our real-life population, with multiple stones in $60.7 \%$ of the cases and a median total stone volume of $1.97 \mathrm{~cm}^{3}$. Patients treated with SMP had single stones in $88.7 \%$ of the cases and a mean diameter of $2.2 \mathrm{~cm} \mathrm{[14].} \mathrm{We} \mathrm{recorded} \mathrm{a} \mathrm{median} \mathrm{OT} \mathrm{of} 90 \mathrm{~min}$, lower than the safety limit of 120 min described in the Literature [16], and a median LT of 28 min. As in the SMP series [14], we observed lower post-operative fever (7.4\%) and transfusion $(0.8 \%)$ rates than reported in the Literature for PCNL (10.8\% and 7\%, respectively) [2]. These results confirm that the principles of mini-invasiveness are respected and that short OTs and controlled IRPs could play a role in the prevention of infectious complications. This may be of extreme relevance in the present scenario characterized by increasingly frequent infections sustained by multi-drugresistant pathogens $[17,18]$. To investigate the association between elevated IRPs and infectious complications, Zhong and colleagues [19] inspected IRPs in-vivo during miniaturized PCNL. They observed that mean IRP $\geq 20 \mathrm{mmHg}$ and accumulative time with IRP $>30 \mathrm{mmHg}$ longer than $50 \mathrm{~s}$ were correlated with post-operative fever. Then, not only IRP peaks can be dangerous for kidney injuries development, but mostly the accumulative time at high pressures can be detrimental for infectious complications. Indeed, elevated IRPs and pyelo-venous backflow are associated with potential systemic absorption of bacteria often colonizing stones and subsequently contaminating the irrigation fluid during lithotripsy. In our series, mean IRP during vmPCNL 
Table 2 Surgery-related variables and post-operative outcomes

\begin{tabular}{|c|c|}
\hline \multicolumn{2}{|l|}{ 2a. Surgery-related variables } \\
\hline & Total: 122 \\
\hline \multicolumn{2}{|l|}{ Laterality $N^{\circ}(\%)$} \\
\hline Right & $55(45.1)$ \\
\hline Left & $67(54.9)$ \\
\hline \multicolumn{2}{|l|}{ Access tract number, $N^{\circ}(\%)$} \\
\hline Single & $111(91)$ \\
\hline Multiple & $11(9)$ \\
\hline \multicolumn{2}{|l|}{ Access tract location, $N^{\circ}(\%)$} \\
\hline Lower calix & $84(68.8)$ \\
\hline Middle calix & $20(16.4)$ \\
\hline Upper calix & $9(7.4)$ \\
\hline Multiple locations & $9(7.4)$ \\
\hline Median total laser energy (IQR), $\mathrm{kJ}$ & $8.63(3,75-16,23)$ \\
\hline \multicolumn{2}{|l|}{ Lapaxy modality, $N^{\circ}(\%)$} \\
\hline Clear Petra Suction & $78(63.9)$ \\
\hline Clear Petra Suction + basket & $44(36.1)$ \\
\hline \multicolumn{2}{|l|}{ Exit strategy, $N^{\circ}(\%)$} \\
\hline Nephrostomy & $97(79.5)$ \\
\hline Ureteric catheter/JJ stent & $5(4.1)$ \\
\hline Nephrostomy + Ureteric catheter /JJ stent & $20(16.4)$ \\
\hline \multicolumn{2}{|l|}{ Intra-operative complications, $N^{\circ}(\%)$} \\
\hline Contrast medium blow-out & $2(1.6)$ \\
\hline Median operative time (IQR), minutes & $90(71-120)$ \\
\hline Median lithotripsy + lapaxy time (IQR), minutes & $28(17-45)$ \\
\hline \multicolumn{2}{|l|}{ 2b. Post-operative outcomes } \\
\hline Median $\mathrm{Hb}$ drop (IQR) g/dL & $1,5(0.6-2.1)$ \\
\hline Transfusions, $N^{\circ}(\%)$ & $1(0.8)$ \\
\hline Complications, $N^{\circ}(\%)$ & $31(25.4)$ \\
\hline Clavien 1 & $14(11.4)$ \\
\hline Clavien 2 & $10(8.2)$ \\
\hline Clavien 3A & $4(3.3)$ \\
\hline Clavien 3B & $3(2.5)$ \\
\hline Median nephrostomy time (IQR), days & $3(2-4)$ \\
\hline Median urethral catheter time (IQR), days & $1(1-1)$ \\
\hline Median length of hospital stay (IQR), days & $4(3-5)$ \\
\hline Stone Clearance Rate, $N^{\circ}(\%)$ & $87(71.3)$ \\
\hline Retreatment, $N^{\circ}(\%)$ & $9(7.4)$ \\
\hline
\end{tabular}

procedures was $13.19 \mathrm{cmH}_{2} \mathrm{O}$ and in no procedure a mean IRP $>27.19 \mathrm{cmH}_{2} \mathrm{O}(20 \mathrm{mmHg})$ was recorded. Alsmadi and colleagues [20], who measured IRPs during SMP, registered an overall average IRP of $19.51 \mathrm{mmHg}\left(26.52 \mathrm{cmH}_{2} \mathrm{O}\right)$ and a mean IRP $>20 \mathrm{mmHg}$ in $29.7 \%$ of the procedures. In both the studies, the threshold of $40.78 \mathrm{cmH}_{2} \mathrm{O}$ was overpassed in most of the procedures (86\% in our series and $79.7 \%$ in Alsmadi's). However, we registered prolonged accumulative time with IRP $>40,78 \mathrm{cmH}_{2} \mathrm{O}$ only in a minority of the cases $(31.8 \%, 22.7 \%$ and $13.6 \%$ for more than $50 \mathrm{~s}, 60 \mathrm{~s}$ and $70 \mathrm{~s}$, respectively) and the median accumulative time with IRP over the threshold was $28.52 \mathrm{~s}$. Alsmadi and colleagues calculated accumulative time with IRP $>40,78 \mathrm{cmH}_{2} \mathrm{O}$ longer than $50 \mathrm{~s}, 60 \mathrm{~s}$ and $70 \mathrm{~s}$ in $36 \%, 32.4 \%$ and $27 \%$ of the cases, respectively, and a median accumulative time with IRP over the threshold of $55 \mathrm{~s}$. The slightly higher IRPs registered during SMP might be due to the use a continuous perfusion pump, that was never applied during the procedures described in the present study, in which irrigation was always provided by gravity, limiting the fluid inflow. 
Table 3 Intrarenal pressures characteristics and fluctuations

3a. IRP characteristics and profile

Mean basic IRP $( \pm \mathrm{SD}), \mathrm{cmH}_{2} \mathrm{O}$

$13.19( \pm 5.99)$

Mean of the mean IRPs during the operations $( \pm \mathrm{SD}), \mathrm{cmH}_{2} \mathrm{O}$

$15.38( \pm 6.24)$

$N$. of cases with peaks of IRP $>40.78 \mathrm{cmH}_{2} \mathrm{O}(30 \mathrm{mmHg}), n$. (\%)

$19(86)$

N. of cases with mean IRP $>27.19 \mathrm{cmH}_{2} \mathrm{O}(20 \mathrm{mmHg}), n$. (\%)

$0(0)$

Median accumulative time with IRP $>40.78 \mathrm{cmH}_{2} \mathrm{O}$ (30 mmHg) (IQR), $\mathrm{s}$

$28.52(12.5-60.04)$

$\mathrm{N}$. of cases with accumulative time (with IRP $>40.78 \mathrm{cmH}_{2} \mathrm{O}$ ) $>50 \mathrm{~s}, n$. (\%)

$7(31.8)$

N. of cases with accumulative time (with IRP $>40.78 \mathrm{cmH}_{2} \mathrm{O}$ ) $>60 \mathrm{~s}, n$. (\%)

$5(22.7)$

N. of cases with accumulative time (with IRP $>40.78 \mathrm{cmH}_{2} \mathrm{O}$ ) $>70 \mathrm{~s}, n$. (\%)

$3(13.6)$

3b. Intrarenal pressure measurements step by step

\begin{tabular}{lll}
\hline Surgical steps & $\begin{array}{l}\text { Overall mean }{ }^{\mathrm{a}} \text { IRP } \\
( \pm \mathrm{SD}), \mathrm{cmH}_{2} \mathrm{O}\end{array}$ & $\begin{array}{l}\mathrm{Overall} \mathrm{max}^{\mathrm{b}} \text { IRP } \\
\left(\mathrm{cmH}_{2} \mathrm{O}\right)\end{array}$ \\
\hline Initial retrograde pyelography & $36.14( \pm 16.75)$ & 65 \\
Renal puncture & $32.46( \pm 12.49)$ & 53,8 \\
Percutaneous pyelography through puncture needle & $37.26( \pm 20.65)$ & 102 \\
Percutaneous tract dilation & $39.10( \pm 18.19)$ & 74 \\
Initial nephroscopy with closed aspiration, max IRP & $39.03( \pm 24.09)$ & 94 \\
Lithotripsy and simultaneous vacuum-lapaxy, mean IRP & $13.29( \pm 6.55)$ & 26.53 \\
Lithotripsy and simultaneous vacuum-lapaxy, max IRP & $28.37( \pm 12.26)$ & 58 \\
Final nephroscopy with closed aspiration, max IRP & $51.15( \pm 11.77)$ & 70 \\
Percutaneous pyelography through access sheath & $53.44( \pm 26.77)$ & 115 \\
Flexible nephroscopy after lithotripsy, max IRP & $19.88( \pm 8.00)$ & 31 \\
Nephrostomy placement & $25.02( \pm 15.37)$ & 64 \\
Final pyelography through nephrostomy tube & $32.07( \pm 21.24)$ & 89 \\
\hline
\end{tabular}

${ }^{a}$ Intended as the mean of the values of the single procedures for the specific surgical step

${ }^{\mathrm{b}}$ Intended as the maximum value reached among the different procedures for the specific surgical step

In our series, among the cases studied for IRP fluctuations, only one patient experienced post-operative fever, thus, it was not possible to identify IRP-related predictive factors for infectious events. This patient had positive pre-operative urine culture and stone culture, treated with targeted fullcourse peri-operative antibiotic therapy. Mean IRP during this procedure was $13.9 \mathrm{cmH}_{2} \mathrm{O}$ and the accumulative time with IRP $>40.78 \mathrm{cmH} 2 \mathrm{O}$ was $15 \mathrm{~s}$, with a maximum peak at $60 \mathrm{cmH}_{2} \mathrm{O}$ during a pyelography (Graphic $\mathrm{n} .5$ in the Online Resource). IRP does not seem to have contributed to postoperative fever in this patient, but the positive cultures, even if treated, represented a risk factor. Potentially, even short times with IRP over the backflow threshold are enough to determine bacterial reabsorption in case of clearly contaminated irrigation fluids.

For what concerns the IRP fluctuations, we observed that during lithotripsy and suction-mediated lapaxy, the threshold of $40.78 \mathrm{cmH} 2 \mathrm{O}$ was overcome in only three procedures (13.6\%) (Graphics n. 9, 11, 12 in the Online Resource), meaning that when aspiration is activated, the risk of uncontrolled pressures is low. During surgical steps, when aspiration is closed, we registered rises in IRP. Maximum pressure peeks were registered during pyelograms in 13 (59\%) procedures, during nephroscopy with closed aspiration in 7 (32\%) and during puncture in $2(9 \%)$. However, the mentioned steps usually are not prolonged in duration, and do not expose the patient to long time elevated IRPs.

The main limitation of this study is the lack of a control group of mini-PCNL procedures performed without aspiration systems which could render our results more significant. One more limitation is represented by the non-uniformity of the imaging modality we adopted in the follow-up: indeed, although CT scan is much more sensitive in identifying residual fragments, in order to reduce the radiation exposure, in particular in recurrent stone formers, we routinely perform US after uneventful procedures performed for small single stones, in which the chance of residual fragments is low. In case of residual fragments at US, a CT scan is performed to plan a retreatment. 


\section{Conclusions}

VmPCNL via the 16 Ch Clear Petra sheath is characterized by a good safety profile and satisfactory stone clearance rates. The most interesting features of this technique are the easy suction-mediated stone fragments removal, the low complications rate and the favorable IRP profile. In particular, in our series, the mean IRP during surgery was always lower than the threshold of pyelo-venous backflow and the accumulative time with IRP over this limit was very short in most of the procedures. The maximal IRP peaks were registered during the surgical steps when aspiration is closed and mostly during pyelograms, whereas, during lithotripsy and vacuum-mediated lapaxy, IRP rarely raised over the threshold.

Author contributions SPZ: protocol development; project development; data collection; data analysis; manuscript writing. EL: project development; data collection; manuscript editing. MF: protocol development; data management; data collection. MT: data collection. AG: manuscript editing. FL: project development. GA: project development. EDL: project development. EM: protocol development, project development, manuscript editing.

Funding The authors received no specific funding for this work.

\section{Compliance with ethical standards}

Conflict of interest The authors declare that they have no conflict of interest.

Ethical approval This study was conducted collecting data obtained for clinical purposes and all the procedures were performed as part of the routine care. Then, our study did not need ethical approval. The procedures used in this study adhere to the tenets of the Declaration of Helsinki.

Informed consent Informed consent was obtained from all individual participants included in the study prior to the procedures.

Open Access This article is licensed under a Creative Commons Attribution 4.0 International License, which permits use, sharing, adaptation, distribution and reproduction in any medium or format, as long as you give appropriate credit to the original author(s) and the source, provide a link to the Creative Commons licence, and indicate if changes were made. The images or other third party material in this article are included in the article's Creative Commons licence, unless indicated otherwise in a credit line to the material. If material is not included in the article's Creative Commons licence and your intended use is not permitted by statutory regulation or exceeds the permitted use, you will need to obtain permission directly from the copyright holder. To view a copy of this licence, visit http://creativecommons.org/licenses/by/4.0/.

\section{References}

1. Turk CSC, Skolarikos A, Neisius A, Petrik A (2019) EAU guidelines. Edn. Presented at the EAU annual congress in Barcelona
2. Seitz C et al (2012) Incidence, prevention, and management of complications following percutaneous nephrolitholapaxy. Eur Urol 61(1):146-158

3. Jackman SV, Docimo SG, Cadeddu JA, Bishoff JT, Kavoussi LR, Jarrett TW (1998) The 'mini-perc' technique: a less invasive alternative to percutaneous nephrolithotomy. World J Urol 16(6):371-374

4. Desai J, Solanki R (2013) Ultra-mini percutaneous nephrolithotomy (UMP): one more armamentarium. BJU Int 112(7):1046-1049

5. Bader MJ, Gratzke C, Seitz M, Sharma R, Stief CG, Desai M (2011) The 'all-seeing needle': initial results of an optical puncture system confirming access in percutaneous nephrolithotomy. Eur Urol 59(6):1054-1059

6. Ganpule AP, Bhattu AS, Desai M (2014) PCNL in the twenty-first century: role of Microperc, Miniperc, and Ultraminiperc. World J Urol 33(2):235-240

7. Wu C, Hua L-X, Zhang J-Z, Zhou X-R, Zhong W, Ni H-D (2017) Comparison of renal pelvic pressure and postoperative fever incidence between standard- and mini-tract percutaneous nephrolithotomy. Kaohsiung J Med Sci 33(1):36-43

8. Doizi S, Uzan A, Keller E, De Coninck V, Rodriauez-Monsalve Herrero M, Traxer O (2018) Comparison of intrarenal pelvic pressure levels during flexible ureteroscopy, minipercutaneous nephrolithotomy and conventional percutaneous nephrolithotomy in a kidney model. Eur Urol Suppl 17(2):e1400-e1401

9. Hinman F (1926) F; Redewill, "pyelovenous back flow". J Am Med Assoc 87:1287-1293

10. Kreydin EI, Eisner BH (2013) Risk factors for sepsis after percutaneous renal stone surgery. Nat Rev Urol 10(10):598-605

11. Charlson ME, Pompei P, Ales KL, MacKenzie CR (1987) A new method of classifying prognostic comorbidity in longitudinal studies: Development and validation. J Chronic Dis 40(5):373-383

12. de la Rosette JJMCH et al (2012) Categorisation of complications and validation of the clavien score for percutaneous nephrolithotomy. Eur Urol 62(2):246-255

13. Singer $\mathrm{M}$ et al (2016) The third international consensus definitions for sepsis and septic shock (Sepsis-3). JAMA 315(8):801

14. Zeng G et al (2016) Super-mini percutaneous nephrolithotomy (SMP): a new concept in technique and instrumentation. BJU Int 117(4):655-661

15. Zeng G, Zhu W, Liu Y, Fan J, Zhao Z, Cai C (2017) The new generation super-mini percutaneous nephrolithotomy (SMP) system: a step-by-step guide. BJU Int 120(5):735-738

16. Xu S et al (2014) A prospective comparative study of haemodynamic, electrolyte, and metabolic changes during percutaneous nephrolithotomy and minimally invasive percutaneous nephrolithotomy. World J Urol 32(5):1275-1280

17. Tandogdu Z, Wagenlehner FME (2016) Global epidemiology of urinary tract infections. Curr Opin Infect Dis 29(1):73-79

18. Foxman B (2014) Urinary tract infection syndromes. Infect Dis Clin North Am 28(1):1-13

19. Zhong W, Zeng G, Wu K, Li X, Chen W, Yang H (2008) Does a smaller tract in percutaneous nephrolithotomy contribute to high renal pelvic pressure and postoperative fever? J Endourol 22(9):2147-2151

20. Alsmadi J, Fan J, Zhu W, Wen Z, Zeng G (2018) The influence of super-mini percutaneous nephrolithotomy on renal pelvic pressure in vivo. J Endourol 32(9):819-823

Publisher's Note Springer Nature remains neutral with regard to jurisdictional claims in published maps and institutional affiliations. 